\title{
P02.196. Changes in medication use associated with Traditional Chinese Medicine for chronic pain
}

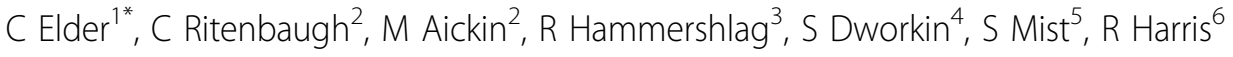 \\ From International Research Congress on Integrative Medicine and Health 2012 \\ Portland, Oregon, USA. 15-18 May 2012
}

\section{Purpose}

A randomized trial of Traditional Chinese Medicine (TCM) for temporomandibular joint dysfunction (TMD) showed a linear decline in pain over 16 TCM visits. Here we investigate whether medication increases could account for improvement, or whether pain medications were also reduced.

\section{Methods}

One hundred sixty-eight TMD patients received TCM or enhanced self-care using a stepped-care design where those who failed on self-care were offered TCM. This report includes 121 patients during their first 16 TCM visits. The initial 8 occurred more often than weekly; patients and practitioners determined subsequent schedules. Outcome data were collected via study-administered questionnaires at standard times, and self-report at every treatment visit. Here we report on average pain (VAS 0-10) and pain medications over the previous week, collected at treatment visits. We converted pain medication intake to equivalent weekly doses of aspirin (for NSAIDs and acetaminophen) or morphine (7.5mg for narcotics). Pain was analyzed by linear regression with random effects for within-individual correlations. Medication use was log-transformed and analyzed using quadratic splines.

\section{Results}

The sample was $85 \%$ female and on average $44(\mathrm{SD}=13)$ years old. Narcotics users' $(n=32)$ average pain improved -2.56 units over 16 visits $(\mathrm{p}<0.001)$. Narcotics use declined until visit $11(-1.73$ doses/wk total, $\mathrm{p}=0.067)$, and then increased to week $16(+1.34$ doses/wk total, $\mathrm{p}=0.076)$. NSAID use declined linearly $(\mathrm{p}=0.019),-0.47$ and -0.20 doses/wk over visits $1-11$ and $11-16$, respectively. For the 18 participants in the top quartile of NSAIDS-only users, average pain decreased linearly over 16 visits $(-1.6$ units, $\mathrm{p}=0.043)$. Dose of NSAIDs declined between visits $1-7(-6.39$ doses/wk, $\mathrm{p}=0.038)$ and increased between visits 7-16 (+1.42 doses/wk, $\mathrm{p}=0.386)$. NSAID use among the rest remained low and stable.

\section{Conclusion}

Among patients using narcotics and those who had the highest NSAID intakes, we observed a short-term reduction in medication use that was partially sustained as $\mathrm{TCM}$ visits became less frequent.

\section{Author details}

${ }^{1}$ Kaiser Permanente Center for Health Research, Portland, USA. ${ }^{2}$ University of Arizona, Tucson, USA. ${ }^{3}$ Oregon College of Oriental Medicine, Portland, USA.

${ }^{4}$ University of Washington, Seattle, USA. ${ }^{5}$ Oregon Health and Science

University, Portland, USA. ${ }^{6}$ University of Michigan, Ann Arbor, USA.

Published: 12 June 2012

doi:10.1186/1472-6882-12-S1-P252

Cite this article as: Elder et al:: P02.196. Changes in medication use associated with Traditional Chinese Medicine for chronic pain. BMC Complementary and Alternative Medicine 2012 12(Suppl 1):P252.

${ }^{1}$ Kaiser Permanente Center for Health Research, Portland, USA

Full list of author information is available at the end of the article

(c) 2012 Elder et al; licensee BioMed Central Ltd. This is an Open Access article distributed under the terms of the Creative Commons 